Case report

\title{
Glycogen storage disease type III presenting with secondary diabetes and managed with insulin: a case report
}

\section{Heba Ismail}

Address: Diabetes, Endocrinology and Metabolism Pediatric Unit, University of Cairo, Egypt

Email: HI - heba2126@yahoo.com

Received: 25 March 2009 Accepted: II June 2009 Published: 17 June 2009

Cases Journal 2009, 2:689| doi: 10.4076/|757-|627-2-689|

This article is available from: http://casesjournal.com/casesjournal/article/view/689 |

(c) 2009 Ismail; licensee Cases Network Ltd.

This is an Open Access article distributed under the terms of the Creative Commons Attribution License (http://creativecommons.org/licenses/by/3.0), which permits unrestricted use, distribution, and reproduction in any medium, provided the original work is properly cited.

\begin{abstract}
Introduction: Reports of secondary diabetes in glycogen storage disease type III have been very limited, where the pathogenesis and management have not been clear. Here we report on a rare case of secondary diabetes in glycogen storage disease type III that has been successfully managed with insulin.

Case presentation: This is a 19-year-old female of Egyptian ethnicity, born of a consanguineous marriage and known to have glycogen storage disease type III since the age of $2 \frac{1}{2}$ years. She presented to us with a history of polyuria, polydipsia, and loss of weight of a few days duration. Physical exam showed stunted growth, hepatomegaly, myopathy and mild dehydration. Emergency labs revealed a fasting blood glucose of $276 \mathrm{mg} / \mathrm{dl}$, but with no ketonuria and arterial blood gases were essentially normal. Her liver transaminases were mildly elevated at the time. Review of her records revealed that the diagnosis of glycogen storage disease type III was made at the age of $2 \frac{1}{2}$ when the mother reported repeated attacks of afebrile (hypoglycemic) convulsions, increasing abdominal girth and failure to thrive. The diagnosis was confirmed by demonstration of debrancher enzyme deficiency on enzymatic assay. Over the years she developed liver dysfunction along with other complications and subsequently her hypoglycemic attacks disappeared a few years prior to her current presentation. After careful consideration of different treatment options, and considering she had been free of hypoglycemic attacks for a few years and had liver dysfunction, we chose to cautiously initiate the patient on insulin therapy. She was still poorly controlled and we gradually increased her total daily dose to $0.8 \mathrm{u} / \mathrm{kg}$. She continued to be free of hypoglycemic attacks and her average daily blood glucose is about $160 \mathrm{mg} / \mathrm{dl}$.
\end{abstract}

Conclusion: We report a rare case of secondary diabetes mellitus in a patient with glycogen storage disease type III managed with insulin. We recommend insulin therapy over oral hypoglycemics to avoid further hepatotoxicity, provided hypoglycemia has resolved. We also recommend serial follow up of glycogen storage disease type III patients with an oral glucose tolerance test for early detection and management of glucose intolerance. 


\section{Introduction}

Glycogen-storage disease type III (GSD III) is an autosomal recessive metabolic disorder caused by loss of function mutations of the glycogen debranching enzyme (Amylo-1,6-glucosidase) gene, located at chromosome band 1p21.2. It is characterized by the storage of abnormal glycogen in both skeletal and cardiac muscle and/or the liver, with variability in resultant organ dysfunction. Major manifestations of GSDIII include hypoglycemia (which may improve and disappear with age), hepatomegaly with elevated transaminases, hyperlipidemia, hyperuricemia, and skeletal myopathy/cardiomyopathy with increased creatine phosphokinase (CPK). There is no specific treatment for GSDs, but diet therapy with nocturnal nasogastric tube feeding and cornstarch improves symptoms (especially hypoglycemia), reduces the liver size, and improves overall growth and development [1]. Rare complications of GSDIII include liver cirrhosis and failure together with secondary glucose intolerance and frank diabetes. However, reports of such cases have been very few. Diabetes mellitus (DM) in GSD III is difficult to treat because patients are prone to hypoglycemia. We, however, report on a case of GSDIII with secondary diabetes successfully controlled with insulin.

\section{Case presentation}

This is a 19-year-old female of Egyptian ethnicity, born of a consanguineous marriage and known to have glycogen storage disease type III since the age of $2 \frac{1}{2}$ years. She presented to us with a history of several days of polyuria, polydipsia, and loss of weight.

Review of her records revealed that she had been diagnosed with GSDIII at the age of $2 \frac{1}{2}$ years, when the mother reported an increasing abdominal girth, failure to thrive, and repeated attacks of afebrile convulsions of 1 year duration. The diagnosis of GSDIII was confirmed at the time by a liver biopsy showing a picture suggestive of GSD (swollen cells and mosaic cytoplasmic appearance with mild fibrosis) as well as demonstration of debrancher enzyme deficiency. She was prescribed long-term therapy with cornstarch for hypoglycemia and colchicine for associated hyperuricemia. Unfortunately, there was variable follow-up by the mother together with non-adherence to management regimens.

Over the years she developed progressive chronic liver dysfunction with persistently high liver transaminases and $\gamma$-glutamyl transpeptidase together with coagulation profile compromise and bleeding tendency. Jaundice was documented only once during an attack of acute hepatitis on top of her chronic dysfunction. Her most recent followup liver biopsy (done years prior to her current presentation) had shown increased fibrosis and changes suggestive of very early cirrhosis, but with intact limiting plates. Subsequently, evidence of vitamin D and A deficiencies developed with metastatic soft tissue calcifications and osteoporotic bone fractures, together with poor night vision and dry skin, respectively. Moreover, the patient later developed myopathy, with progressive muscle weakness and pain, indicating a diagnosis of GSDIII type a (both liver and muscle involvement). Fortunately, about four years prior to her current presentation the patient's hypoglycemic attacks improved and eventually disappeared. Her family history is positive for two younger sibs, both diagnosed with GSD.

Upon physical exam, she appeared dysmorphic, stunted, with a mild degree of dehydration and dry skin. Abdominal exam revealed a firm and enlarged liver, but with no splenomegaly or ascites. Neuromuscular system examination revealed reduced muscle power in both upper and lower limbs, while cardiovascular system exam excluded any evidence of cardiomyopathy. Pubertal staging revealed primary amenorrhea, but with adequate development of secondary sex characters. Positive clinical findings are summarized in Table 1.

Emergency labs done at the time of presentation revealed a fasting blood glucose (FBG) of $276 \mathrm{mg} / \mathrm{dl}$ and no ketones. Her arterial blood gases (ABG) were essentially normal with a $\mathrm{pH}$ of 7.36, $\mathrm{PCO}_{2} 44.1, \mathrm{HCO}_{3} 22.1, \mathrm{BE}-1.1$.

Further lab studies showed pus cells in urine, and a culture later grew Escherichia coli. A complete blood count with a differential count showed a marked shift to the left. Her liver transaminases, $\gamma$-glutamyl transpeptidase and alkaline phosphatase were mildly elevated, while her kidney functions and electrolytes were normal. Her uric acid and lactate levels were also normal, but her creatine phosphokinase was high at the time.

After careful consideration of different treatment options and considering the patient was free of hypoglycemic attacks for an adequate time without treatment and that she had already developed liver dysfunction and early cirrhosis, we decided to begin the patient on very low doses of insulin therapy. She continued to show high blood glucose (BG) readings with a daily average of about $263 \mathrm{mg} / \mathrm{dl}$, requiring that we gradually increase her total

Table I. Clinical presentation of our case

\begin{tabular}{ll}
\hline Physical Examination & Positive Findings \\
\hline General appearance & Facial dysmorphism and stunted growth \\
Anthropometry & Height -2.2 SDS, Weight -4 SDS \\
Skin & Dry skin and mild dehydration \\
Abdominal & Liver $\approx 5 \mathrm{~cm}$ below the costal margin and firm \\
Neuromuscular & Bilateral and symmetrically reduced muscle \\
& power $(4 / 5)$ in both upper and lower limbs \\
\hline
\end{tabular}




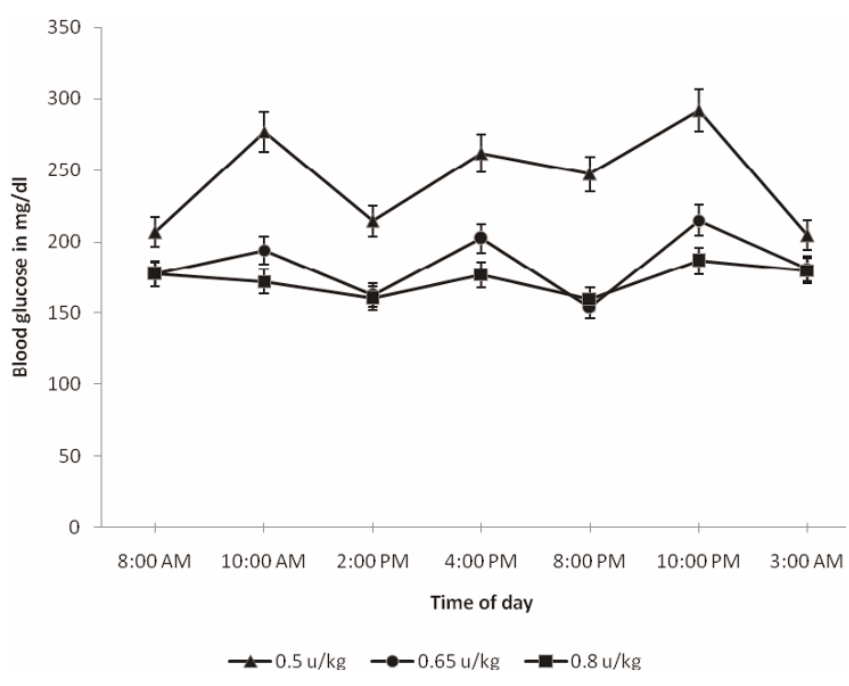

Figure I. Daily blood glucose levels on different insulin doses.

dose to about $0.8 \mathrm{u} / \mathrm{kg} /$ day. She is currently relatively well controlled and has continued to be free of hypoglycemic attacks; rather she tends to err on the high side with a daily average BG of approximately $160 \mathrm{mg} / \mathrm{dl}$. Figure 1 shows average daily glucose levels during the initial period of dose adjustment.

\section{Discussion}

Reports on the pathogenesis of secondary diabetes in GSDIII and its management have been very limited and relatively unclear $[2,3]$. Glucose intolerance and secondary diabetes in GSDIII is thought to result from liver dysfunction. The pathogenesis of glucose intolerance in liver dysfunction in general also remains unclear, where several factors are thought to be responsible, including insulin resistance, insulin hypersecretion with reduced clearance, impaired glycogen catabolism, defective adipocyte insulin sensitivity and others. Diabetes development in patients with liver cirrhosis, regardless of etiology, shows that about $80 \%$ develop glucose intolerance, while up to $40 \%$ can develop frank DM [4,5]. Early on there is post-prandial hyperglycemia (PPHG), but later on there is both fasting \& PPHG. Of note here is that our patient had already developed liver dysfunction and early cirrhosis at the time she presented to us. She was unfortunately missed during the earlier stages of intolerance and PPHG due to her loss to follow up.

Classically, DM associated with liver cirrhosis is treated with insulin; however, this becomes a major problem if cirrhosis is secondary to GSDIII due to the high risk of hypoglycemia. Oki et al. [3] described an adult GSDIII patient who later developed liver dysfunction. This dysfunction worsened over the years, and he subsequently developed PPHG. Being that this patient presented with PPHG, the authors chose to treat him with an $\alpha$-glucosidase inhibitor, voglibose. Alpha-glucosidase inhibitors blunt PPHG by essentially delaying the hydrolysis of carbohydrates in the intestines. Accordingly, there is a low risk for hypoglycemia. However, when this option was considered in our patient, it was thought unhelpful given that she had presented to us at a more advanced stage of fasting hyperglycemia. Furthermore, there has been conflicting evidence regarding the potential hepatotoxicity of voglibose [6-8]. So considering our case had elevated liver transaminases, we chose not to take this risk.

Due to the patient's presentation with fasting hyperglycemia, the spontaneous resolution of her hypoglycemic attacks for several years prior to clinical diabetes, and the potential hepatotoxic effect of most oral hypoglycemics, we decided to manage her diabetes with insulin therapy and have been so far successful.

\section{Conclusion}

We report a rare case of secondary DM in a GSDIII patient who has been successfully managed with insulin. We recommend insulin therapy over oral hypoglycemics to avoid further hepatotoxicity, provided hypoglycemias have resolved. We also recommend serial follow up of GSDIII patients with an oral glucose tolerance test (OGTT) for early detection and management of glucose intolerance.

\section{Abbreviations}

BG, Blood glucose; DM, Diabetes Mellitus; GSD, Glycogen storage disease; GSDIII, Glycogen storage disease type III; OGTT, Oral glucose tolerance test; PPHG, Post-prandial hyperglycemia; SDS, Standard deviation score.

\section{Consent}

Written informed consent was obtained from the patient's mother for publication of this case report. A copy of the written consent is available for review by the Editor-inChief of this journal.

\section{Competing interests}

The author declares that she has no competing interests.

\section{Author contribution}

HI provided initial management and follow up of this case's diabetes.

\section{Acknowledgements}

Special thanks to Professors Dr. Mona Hafez and Dr. Mona Mamdouh for their advice and consultations regarding the management of diabetes in this case. Special thanks also to Assistant Professor Dr. Mona El-Razky for providing information regarding the patient's hepatic condition, diagnosis and management. 


\section{References}

I. Smit GPA, Rake JP, Akman HO, DiMauro S: Glycogen Storage Disease Type III (Debranching Enzyme Deficiency). In: Inborn Metabolic Diseases: Diagnosis and Treatment. 4th edition. Edited by John Fernandes, Jean-Marie Saudubray, Georges Van Den Berghe, John H. Walter. Springer; 2006:108-109.

2. Moe PJ, Waaler PE, Garatun-Tjeldstù $O$ : Glycogen storage disease type III and diabetes mellitus. Acta Paediatr Scand 1972, 61:483486.

3. Oki Y, Okubo M, Tanaka S, Nakanishi K, Kobayashi T, Murase T: Diabetes mellitus secondary to glycogen storage disease type III. Diabet Med 2000, I7:8I0-8I2.

4. Collins JR, Crofford OB: Glucose intolerance and insulin resistance in patients with liver disease. Arch Intern Med 1969, 124: I42-148.

5. Conn HO, Schreiber W, Elkington SG: Cirrhosis and diabetes. II. Association of impaired glucose tolerance with portalsystemic shunting in Laennec's cirrhosis. Am J Dig Dis 1971, 16:227-239.

6. Masumoto T, Ishikawa M, Yamauchi Y, Hiasa Y, Yamamoto $K$, luchi $H$, Ohkubo K, Akbar SMF, Michitaka K, Horiike N, Onji M: Druginduced hepatitis with severe cholestasis due to voglibose. International Hepatology Communications 1996, 5:289-296.

7. Shuichi, Takushi A, Kouji H, Hirotaka M, Makoto O, Hiroomi M, Terukatsu A: Hepatic Necrosis with Cholestasis Induced by Long-term Voglibose Administration. Internal Medicine 200I, 40:484-488

8. Goke B, Fuder $H$, Wieckhorst G, Theiss $U$, Stridde E, Littke T, Kleist P, Arnold R, Lücker PW: Voglibose (AO-I 28) is an efficient alpha-glucosidase inhibitor and mobilizes the endogenous GLP-I reserve. Digestion I955, 56:493-50I.

\section{Do you have a case to share?}

Submit your case report today

- Rapid peer review

- Fast publication

- PubMed indexing

- Inclusion in Cases Database

Any patient, any case, can teach us something

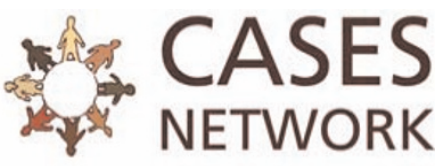

www.casesnetwork.com 